1 Department of Oral Pathology, Saveetha Dental College and Hospitals, Saveetha Institute of Medical and Technical Sciences, Saveetha University.

Corresponding author:

Snega Thamilselvan,

Saveetha Dental College \& Hospitals,

Saveetha Institute of Medical And

Technical Sciences, Saveetha

University,

162, Poonamallee High Road,

Velappanchavadi,

Chennai - 600077

Tamilnadu, India.

Snegathamilselvann@gmail.com

Phone number: 7397458628

Email Id-151907001.Sdc@

Saveetha.Com

Editor:

Dr Altair A. Del Bel Cury

Received: July 1 1', 2021

Accepted: February 2, 2021

\section{p53 \& Cyclin D1 expression in surgically resected clear margins of oral squamous cell carcinoma}

\author{
Snega Thamilselvan ${ }^{1, *}$ iD, Archana Santhanam ${ }^{1}$ (iD, \\ Herald J. Sherlin ${ }^{1}$ (ID), Gifrina Jayaraj ${ }^{1}$ (iD, K. R. Don ${ }^{1}$ (iD
}

Oral squamous cell carcinoma (OSCC) is one of the most well-known malignancies that affect the human population worldwide. The early diagnosis and early intervention of OSCC help improve the survival rate of the patients. The tumour free surgical margins are a positive prognostic factor for recurrence-free survival. The molecular markers can be used to detect the tumour free surgical margins. Aim: The aim of the study is to evaluate the expression of p53 \& Cyclin D1 marker in resected surgical apparently clear margins and to correlate the p53 \& Cyclin D1 expression with clinicopathological characteristics and patient outcome. Methods: The study population included retrospective cases of OSCC with apparently clear margins (2017-18) n=10 and Clinicopathological variables relevant to survival analysis were recorded. Finally, two margins were selected from each case, a total of 20 margins were included in this study. Paraffin-embedded wax blocks retrieved and tissue sections were made. Expression of cyclin D1 and p 53 was assessed by the immunohistochemical staining procedure Results: Positive expressions Cyclin D1 in $40 \%$ of mild dysplasia margins and $60 \%$ in clearance adequate margins were present. p53 expression was seen in 16\% of mild dysplasia margins and $84 \%$ in clearance adequate margins. The expression of p53 and Cyclin D1 molecular markers are noted in the basal \& parabasal layer of epithelium. Conclusion: Molecular markers could play a more reliable method for the assessment of dysplasia at the margins.

Keywords: Tumor suppressor protein p53. Cyclin D1. Carcinoma, squamous cell. 


\section{Introduction}

Oral cancer makes up to $2 \%$ of all the cancer cases with the majority being Oral Squamous Cell Carcinoma (OSCC) which accounts for $90 \%$ of all the oral malignancies ${ }^{1}$. Oral cancer is the 8th most frequent cancer among males and the 14th most frequent cancer among females globally². The current findings state the increasing prevalence of oral cancer in Asia, especially in India has been documented'.

Surgical resection is the first-line management of OSCC followed by adjuvant radiotherapy and chemotherapy when needed ${ }^{3}$. The primary goal of surgical resection is to obtain tumour-free margins. The tumour-free surgical margin is an important prognostic factor for recurrence free survival in OSCC managed with primary surgery 4 . Regardless of whether the histological status of surgical margins is apparently clear the local recurrence rate of OSCC still ranges from 10\% - 30\% ${ }^{4}$. The severe dysplasia margins are considered positive margin which requires a re-excision while mild/moderate dysplasia margins are being overseen leading to local recurrence.

Head and Neck Squamous Cell Carcinoma (HNSCC) is a multistep process characterized by genetic and epigenetic alterations. These alterations in the tumour-free surgical margins that lead to recurrence may not be detected by conventional microscopic histological analysis but may be detected using immunohistochemical (IHC) staining ${ }^{5,6}$

TNM staging and histopathological grading are considered as the main prognostic factors in OSCC. But patients with similar stages of disease treated in a uniform manner experience a wide range of outcomes. The biological behavior of cancer for each patient differs ${ }^{7}$, which necessitates assessing the molecular markers separately and according to which the treatment modalities can be tailor-made.

OSCC is characterized by imbalances in cell cycle control. The assessment of p53 \& Cyclin D1 molecular markers in surgical margins is more valuable in surgical margins for patients undergoing surgical treatment. p53 is a gene that codes for a protein that regulates cell growth and proliferation through its role in cell-cycle checkpoint control hence functions as a tumour suppressor ${ }^{5}$. The Cyclin D1 is a proto-oncogene that encodes the Cyclin D1 nuclear protein, a positive regulator of G1 cell-cycle checkpoint and may play an important role in tumorigenesis of OSCC ${ }^{8}$. Therefore, expression of p53 \& overexpression of Cyclin D1 in the resected surgical apparently clear margins is considered to have better prognostic value in OSCC. This study evaluates the expression of p53 \& CyclinD1 markers in resected surgical apparently clear margins and to correlate the p53 \& CyclinD1 expression with clinicopathological characteristics and patient outcome.

\section{Materials and methods}

\section{Sample Selection}

A total of 40 retrospective cases of OSCC patients who reported to Saveetha Dental College \& Hospitals from 2017-2018 were selected initially. Clinic-pathological variables relevant to survival analysis were recorded. All the patients had been treated 
surgically and the margins of the excised specimens were histopathologically evaluated for adequate clearance. 30 retrospective cases were excluded since the surgical margins had moderate to severe dysplasia histopathologically, inadequate thickness of the epithelium, fragmented tissue sections and also the patients who underwent adjuvant chemotherapy and radiotherapy in follow-up were excluded. A final of 10 cases that were reported with adequate epithelial thickness and apparently clear or mild dysplasia margins were included in the study. 2 margins from each case were included in the study. Finally, the histological analysis for the respective slides was reviewed and with a total of 20 margins, the study was performed. Approval for the study was obtained from the Institutional Review Board SRB/SDC/MDS/002/03.

\section{Immunohistochemistry}

The paraffin wax blocks were retrieved from the Department of Oral \& Maxillofacial Pathology from Saveetha Dental College \& Hospital. $3 \mu \mathrm{m}$ sections were cut from formalin-fixed paraffin-embedded blocks mounted on gelatin-coated slides. Then sections were deparaffinized in xylene for 10 mins \& followed by dehydration in 100\% alcohol for 5 mins and rinsed in distilled water. Following which heat mediated antigen retrieval with Tris-EDTA buffer solution of $9.0 \mathrm{pH}$ was done in a pressure cooker for 5 mins. Depressurize the pressure cooker to $37 \mathrm{c}$ under running tap water. Endogenous peroxidase was blocked for 30 mins. Sections were incubated with the primary antibody, p53 (Dako, Monoclonal mouse anti-human p53 protein, Denmark) \& cyclin D1(Dako, Monoclonal mouse anti-human cyclin D1, Denmark) for 1 hour at room temperature. Detection was performed using polyexcel HRP/DAB detection system (Pathnsitu, conjugated by goat anti-mouse/rabbit IgG, USA). The sections were then counterstained with Mayer's hematoxylin and were then dehydrated and mounted using dibutyl phthalate in xylene mountant. Negative and positive controls were used in each run.

\section{Scoring criteria}

The presence of brown-coloured reactions at the site of the target antigen was indicative of positive reactivity. The parameters used for assessing the immunostaining was propensity index, which indicates the percentage of tumour cells which had taken up the stain and staining intensity, which indicates the amount of stain taken up by the tumour cells. Immunostaining was assessed by the evaluation of a total score obtained by combining the staining intensity and staining proportion scores of p 53 and cyclin -D1 cells which were scored from 0 to $3+$. The scores for evaluation of immunostaining are tabulated in table 1. There were 2 observers who assessed and evaluated the respective slides for immunohistochemical analysis.

Table 1. Scoring criteria for evaluation of expression of p53 and Cyclin D1.

\begin{tabular}{lcc}
\hline Score & Proportionality index & Intensity of Staining \\
\hline 0 & No labelling or $<10 \%$ of tumour cells & negative \\
\hline $1+$ & $10-24 \%$ of tumour cells & mild \\
\hline $2+$ & $25-49 \%$ of tumour cells & moderate \\
\hline $3+$ & $>50 \%$ of tumour cells & severe \\
\hline
\end{tabular}




\section{Statistical analysis}

All the results were tabulated and assessed for statistical analysis using SPSS (IBM SPSS Statistics for Mac Version 20.0). The results of the two markers were compared using the Chi-square test, and $p$-value $=0.05$ was statistically significant. The Kaplan-Meier method was used to estimate local recurrence-free survival and the statistical significance was determined by the log-rank test.

\section{Results}

All the resected surgical margins of $n=10$ OSCC cases included in the study were histopathologically clear/mild dysplasia margins. A total of $n=10$ cases with 2 margins for each were selected and analyzed for immunohistochemistry staining to evaluate the expression of $\mathrm{p} 53$ and Cyclin D1. Among 20 margins evaluated for IHC expression $6(30 \%)$ were mild dysplasia margins and $14(70 \%)$ were apparently clear margins.

Table 2 shows patient characteristics. Pathologically, $80 \%$ of the patients had metastasis of which $30 \%$ involved level I, 40\% involved level II \& 10\% involved level IV and $20 \%$ of the patients had no metastasis. Out of 10 cases, 2 cases( $20 \%$ ) had a recurrence and the survival rate was $90 \%$ among 10 patients.

Table 2. Table depicting the demographics, tumour characteristics and the overall survival of the patients included in the study.

\begin{tabular}{|c|c|c|}
\hline Factor & Group & Total sample N(\%) \\
\hline \multirow[t]{2}{*}{ Gender } & Male & $8(80 \%)$ \\
\hline & Female & $2(20 \%)$ \\
\hline \multirow[t]{2}{*}{ Age } & $>50$ & $5(50 \%)$ \\
\hline & $<50$ & $5(50 \%)$ \\
\hline \multirow[t]{6}{*}{ Location } & Lateral border of the tongue & $3(30 \%)$ \\
\hline & Left buccal mucosa & $3(30 \%)$ \\
\hline & Right buccal mucosa & $1(10 \%)$ \\
\hline & Left maxilla & $1(10 \%)$ \\
\hline & Gingivobuccal sulcus & $1(10 \%)$ \\
\hline & Palate & $1(10 \%)$ \\
\hline \multirow[t]{5}{*}{ Nodal status } & $L-I$ & $3(30 \%)$ \\
\hline & $L-I I$ & $4(40 \%)$ \\
\hline & L- III & 0 \\
\hline & $L-I V$ & $1(10 \%)$ \\
\hline & No involvement & $2(20 \%)$ \\
\hline \multirow[t]{4}{*}{ Histological grade } & Microinvasion & $1(10 \%)$ \\
\hline & WDSCC & $7(70 \%)$ \\
\hline & MDSCC & $1(10 \%)$ \\
\hline & PDSCC & $1(10 \%)$ \\
\hline \multirow[t]{2}{*}{ Recurrence } & yes & $2(20 \%)$ \\
\hline & No & $8(80 \%)$ \\
\hline \multirow[t]{2}{*}{ Survival } & Alive & $9(90 \%)$ \\
\hline & Expired & $1(10 \%)$ \\
\hline
\end{tabular}


Cyclin D1 expression in resected surgical margins showed positive expression in 5 (25\%) margins. Out of the 5 margins, 2 (40\%) margins were mild dysplasia margins and $3(60 \%)$ were clearance adequate margins. There were negative expressions of cyclin D1 in 15 margins (75\%) with 4 (27\%) in mild dysplasia margins and 11 (73\%) in clearance adequate margins. The level of expression of cyclin D1 was seen in the basal and parabasal layers of the epithelium (Table 3). The positive margins of cyclin D1 in basal and parabasal layers are depicted in figure 1.

Table 3. Expression of cyclin D1 in the resected surgical apparently clear margins.

\begin{tabular}{cccc}
\hline Margins & Positive expression $(\mathbf{n}=5)$ & Negative expression $(\mathbf{n}=15)$ & Level of expression \\
\hline Mild dysplasia & $2(40 \%)$ & $4(27 \%)$ & Basal \& parabasal \\
\hline Clearance adequate & $3(60 \%)$ & $11(73 \%)$ & Basal \& parabasal \\
\hline
\end{tabular}

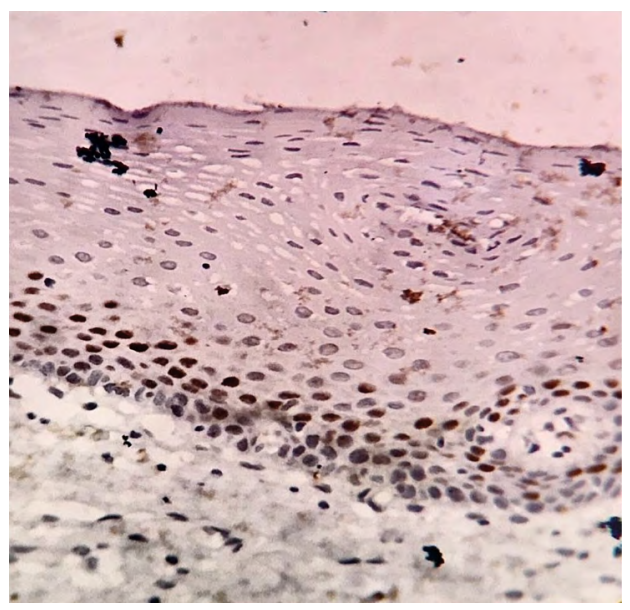

Figure 1. Positive expression of cyclin D1 in the basal and parabasal layer of resected surgical margins.

Among the 20 margins evaluated for p53 expression, positive expression was present in 12 (60\%) margins. Out of the 12 margins, 2 (16\%) margins were mild dysplasia margins and 10 (84\%) were clearance adequate margins. There were negative expressions of p53 in 8 (40\%) margins with 4 (50\%) in mild dysplasia margins and 4 (50\%) in clearance adequate margins. The level of expression of p53 was seen in the basal and parabasal layers of the epithelium (Table 4) (figure 2).

Table 4. Expression of p53 according in the resected surgical apparently clear margins.

\begin{tabular}{cccc}
\hline Margins & Positive expression $(\mathbf{n}=\mathbf{1 2})$ & Negative expression $(\mathbf{n}=\mathbf{8})$ & Level of expression \\
\hline Mild dysplasia & $2(16 \%)$ & $4(50 \%)$ & Basal \& Parabasal \\
\hline Clearance adequate & $10(84 \%)$ & $4(50 \%)$ & Basal \& Parabasal \\
\hline
\end{tabular}




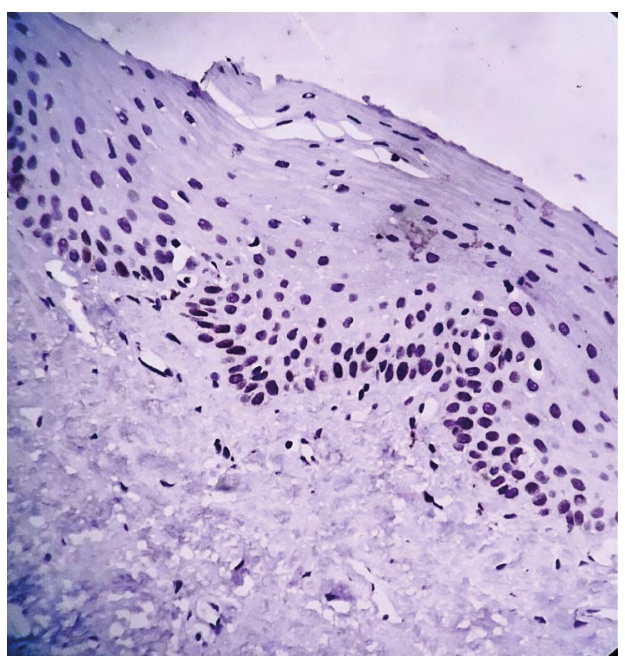

Figure 2. Positive expression of p53 in the basal and parabasal layers of resected surgical margins.

Among the 20 margins, 2 (10\%) clearance adequate margins of one case showed positive expression for both p53 and cyclin D1. The level of expression of p53 and cyclin D1 was seen in basal and parabasal layers of the epithelium.

The Kaplan-Meier survival local recurrence-free survival curve according to the IHC status in surgical margins showed patients with cyclin D1 positive expression in surgical margins are $100 \%$ alive, $80 \%$ of cyclin D1 negative expressions in surgical margins are alive and 20\% with cyclin D1 negative expression in the surgical margin are deceased. The $p$-value $=0.373(p>0.050)$ (figure 3$)$.

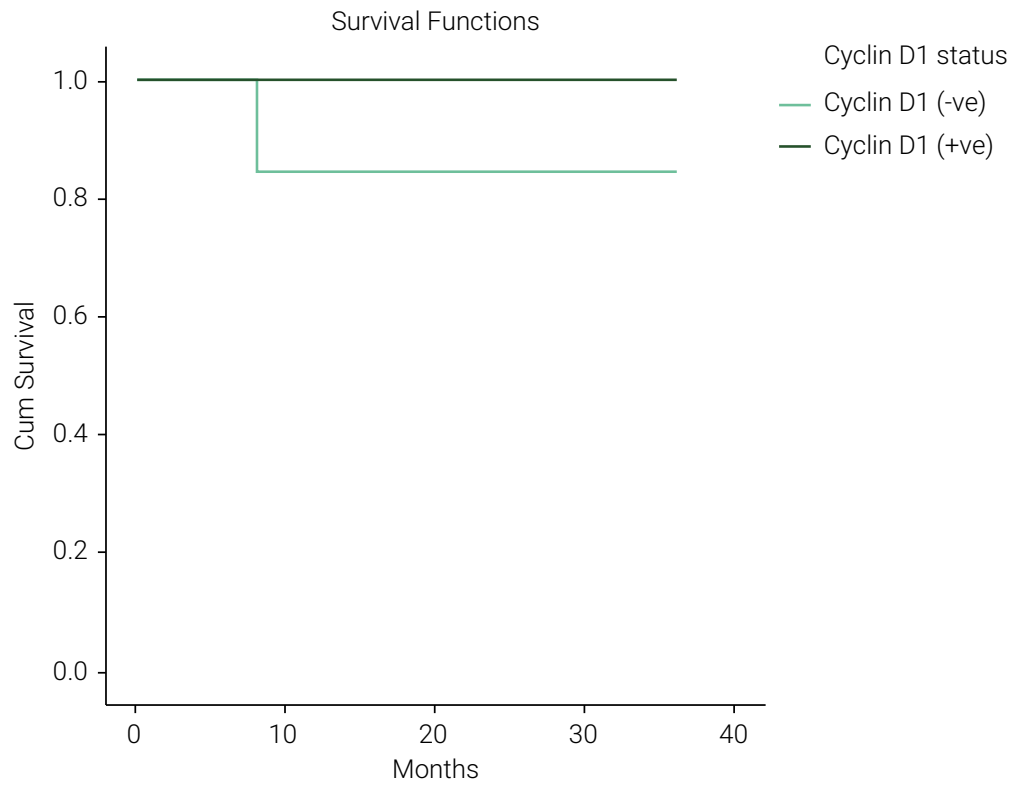

Figure 3. Cumulative local recurrence free survival curve for cyclin D1 expression in resected surgical margins 
The Kaplan-Meier survival local recurrence-free survival curve according to the IHC status in surgical margins showed patients with p53 negative expressions in surgical margins are $100 \%$ alive, $80 \%$ of p53 positive expressions in surgical margins are alive and $20 \%$ with positive expression in the surgical margin is deceased. The p-value $=0.665(p>0.05)$ (figure 4).

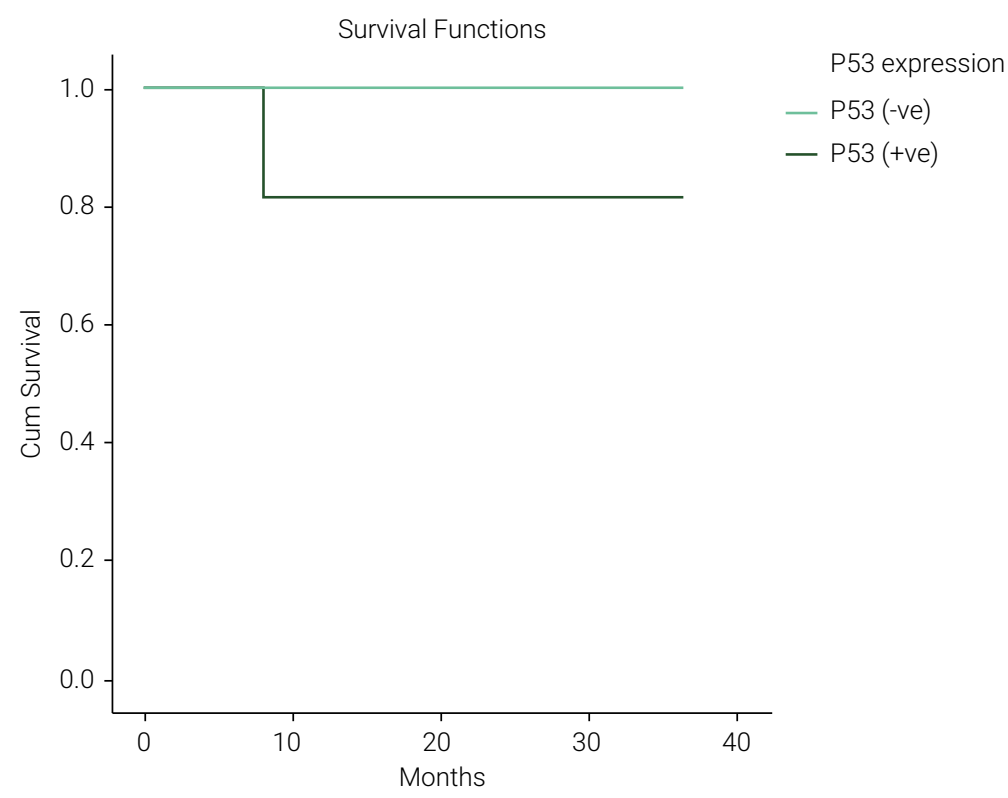

Figure 4. Cumulative local recurrence free survival curve for p53 expression in resected surgical margins.

\section{Discussion}

Immunohistochemistry $(\mathrm{IHC})$ is an integration of histological and immunological techniques that mainly visualizes the distribution and localization of specific molecular biomarkers within a tissue ${ }^{9}$. IHC staining has an important role in the histopathological diagnosis of many tumours ${ }^{10}$. A local recurrence after resection indicates the presence of molecular alterations in cells. p53 is a classical tumour suppressor gene, its expression is related to the tumorigenesis and overexpression of cyclin D1 represents the same. The pathological evaluation of oral epithelial dysplasia is based on the epithelial architectural and cellular features and is graded accordingly ${ }^{4}$. Severe dysplasia has been considered as positive margins that require re-excision after histopathological evaluation. The mild/ moderate dysplasia margins are usually overlooked which indicates the need for evaluation of molecular markers to avoid recurrence. To date, there have been several studies done with the primary tumour specimen or invasive tumour front for evaluating the expression of molecular biomarkers and prognosis in oral squamous cell carcinoma patients. However, the analysis of molecular markers in resected surgical margins would be more appropriate in determining the prognosis and survival of the patients. To the best of our knowledge, this is the first study to evaluate p53 and cyclin D1 expression in resected surgical margins. 
The positive expressions of p53 were seen in 12 margins out of the total 20 margins of which 10 margins were clearance adequate margins and 2 were mild dysplasia margins. Interestingly, in patients who had clear margins, a majority of the patients who had p53 positive expression did not develop local recurrence. Only one patient developed local recurrence at the end of five months and another patient with positive p53 expression expired after 8 months. Also, the statistical significance of the overall survival rate with the positive expression of p53 was not significant ( $p$-value $=0.665$ ). Since the level of expression for p53 was restricted to basal and parabasal layers, it cannot be accepted as a confirmatory prognostic indicator yet it adds value for existing cancer with respect to local recurrence. The previous literature suggests the presence of a strong impact of p53 on local recurrence. Also, they have observed p53 expression in the late event of carcinogenesis ${ }^{11}$. Use of immunohistochemistry alone to determine whether the positive p53 expression reflects the presence of stable mutant p53 protein or stabilization of normal p53 through its binding to certain cellular gene products is not sufficient. On the other hand, there will be false-negative staining for p53 when the nonsense and frame-shift mutations result in the absence of p53 in the tumour cells. Therefore, p53 immunoexpression does not exactly correspond with the p53 gene status ${ }^{12}$.

Our study results showed positive expression of cyclin D1 in 5 margins of which 3 were clearance adequate margins and 2 were mild dysplasia margins. All the patients with positive cyclin D1 expression in surgical margins were alive and disease-free. The cyclin D1 positivity in surgical margins did not have any significance in our study because carcinogenesis is multifactorial with the involvement of numerous genes and pathways. The low-level expression of cyclin D1 is typical of epithelial cells in a normal state as a cell cycle regulator in the G1-S phase transition. Cyclin D1 overexpression in surgical margins may cause future carcinogenesis ${ }^{13}$. Overexpression of cyclin D1 has been previously reported in many malignancies such as breast cancer, colon cancer, prostate cancer, lymphomas, melanomas and carcinomas $^{13,14}$. Sakashita et al. ${ }^{5}$ identified cyclin D1-positive tumour specimens did not indicate a worse prognosis, but cyclin D1-positive margins could be a worse prognostic factor for local recurrence. The presence of cyclin D1-positive surgical margins did not have any statistical significance on overall survival $(p=0.373)$. Hence, cyclin D1-positive status in surgical margins can be considered as an unbiased prognostic indicator for local recurrence.

In the present study, the positive expression of both the molecular markers, p53 and cyclin D1 was noted in one case in both the margins. Both the margins were clear margins. Unfortunately, the positive expression does not prove any correlation with the prognosis since the patient is alive and disease-free.

The overall survival of OSCC patients is determined by several factors such as age, gender, T and N stage, tumour differentiation, primary site, multiple nodal metastases, extracapsular spread, massive primary cancer and the presence of adjunctive treatment ${ }^{15}$. Local recurrence is observed less frequently in patients with histopathologically tumour-free surgical margins. The retrospective analysis was done with a minimum sample size selected from paraffin-embedded wax blocks. Hence, large scale retrospective studies are required to substantiate the results obtained. 
A clear surgical margin is an important determinant of a good outcome. In the present study, some patients with clear margins developed local recurrence while some patients did not. IHC analysis of surgical margins can augment standard histopathological assessment and may improve the prediction of local recurrence. These data may have a major impact on future diagnostic workups for patients with oral carcinoma after surgical treatment.

In conclusion, molecular markers could play a more reliable method for the assessment of dysplasia at the margins. Further large scale studies to examine the association between p53 and Cyclin D1 expression at the margin of OSCC and the development of local recurrence are required.

\section{Acknowledgements}

The authors would like to acknowledge the help and support rendered by the Department of Oral Pathology of Saveetha Dental College and Hospitals and the management for their constant assistance with the research.

\section{References}

1. Ghafari R, Jalayer Naderi N, Emami Razavi A. A retrospective institutional study of histopathologic pattern of Oral Squamous Cell Carcinoma (OSCC) in Tehran, Iran during 2006-2015. J Res Med Sci. 2019 Jun;24:53. doi: 10.4103/jrms.JRMS_882_18.

2. Coelho KR. Challenges of the oral cancer burden in India. J Cancer Epidemiol. 2012;2012:701932. doi: 10.1155/2012/701932.

3. Kamat M, Rai BD, Puranik RS, Datar UV. A comprehensive review of surgical margin in oral squamous cell carcinoma highlighting the significance of tumor-free surgical margins. J Cancer Res Ther. 2019 Jul-Sep;15(3):449-54. doi: 10.4103/jcrt.JCRT_273_17.

4. Yang XH, Ding L, Fu Y, Chen S, Zhang L, Zhang XX, et al. p53-positive expression in dysplastic surgical margins is a predictor of tumor recurrence in patients with early oral squamous cell carcinoma. Cancer Manag Res. 2019 Feb;11:1465-72. doi: 10.2147/CMAR.S192500.

5. Sakashita T, Homma A, Suzuki S, Hatakeyama H, Kano S, Mizumachi T, et al. Prognostic value of cyclin D1 expression in tumor-free surgical margins in head and neck squamous cell carcinomas. Acta Otolaryngol. 2013 Sep;133(9):984-91. doi: 10.3109/00016489.2013.795287.

6. Bilde A, von Buchwald C, Dabelsteen E, Therkildsen MH, Dabelsteen S. Molecular markers in the surgical margin of oral carcinomas. J Oral Pathol Med. 2009 Jan;38(1):72-8. doi: 10.1111/j.1600-0714.2008.00715.x.

7. Sheriff K, Santhanam A. Knowledge and awareness towards oral biopsy among students of Saveetha Dental College. Res J Pharm Technol. 2018;11(2):543-6. doi: 10.5958/0974-360X.2018.00101.4.

8. Zhong LP, Zhu DW, William WN Jr, Liu Y, Ma J, Yang CZ, et al. Elevated cyclin D1 expression is predictive for a benefit from TPF induction chemotherapy in oral squamous cell carcinoma patients with advanced nodal disease. Mol Cancer Ther. 2013 Jun;12(6):1112-21. doi: 10.1158/1535-7163.MCT-12-1013.

9. Duraiyan J, Govindarajan R, Kaliyappan K, Palanisamy M. Applications of immunohistochemistry. J Pharm Bioallied Sci. 2012 Aug;4(Suppl 2):S307-9. doi: 10.4103/0975-7406.100281.

10. Hornick JL. Novel uses of immunohistochemistry in the diagnosis and classification of soft tissue tumors. Mod Pathol. 2014 Jan;27 Suppl 1:S47-63. doi: 10.1038/modpathol.2013.177. 
11. Singh J, Jayaraj R, Baxi S, Mileva M, Skinner J, Dhand NK, et al. Immunohistochemical expression levels of p53 and elF4E markers in histologically negative surgical margins, and their association with the clinical outcome of patients with head and neck squamous cell carcinoma. Mol Clin Oncol. 2016 Feb;4(2):166-72. doi: 10.3892/mco.2015.689.

12. Sawair F, Hassona Y, Irwin C, Stephenson M, Hamilton P, Maxwell P, et al. p53, Cyclin D1, p21 (WAF1) and Ki-67 (MIB1) Expression at Invasive Tumour Fronts of Oral Squamous Cell Carcinomas and Development of Local Recurrence. Asian Pac J Cancer Prev. 2016;17(3):1243-9. doi: 10.7314/apjcp.2016.17.3.1243.

13. Fu M, Wang C, Li Z, Sakamaki T, Pestell RG. Minireview: Cyclin D1: normal and abnormal functions. Endocrinology. 2004 Dec;145(12):5439-47. doi: 10.1210/en.2004-0959.

14. Lukas J, Bartkova J, Rohde M, Strauss M, Bartek J. Cyclin D1 is dispensable for G1 control in retinoblastoma gene-deficient cells independently of cdk4 activity. Mol Cell Biol. 1995 May; 15(5):2600-11. doi: 10.1128/mcb.15.5.2600.

15. Shehenaz A, Santhanam A, Jayaraj G, Ramani P. Correlation of levels of lymph node, node involvement, gender predilection and histopathology in surgical pathology specimen of oral squamous cell carcinoma. Int J Res Trends Innov. 2017;2(8):149-53. 